\title{
A NOTE ON LOW ENERGY SCATTERING FOR HOMOGENEOUS LONG RANGE POTENTIALS
}

\author{
RUPERT L. FRANK
}

The very interesting, recent paper DeSk2] is concerned with scattering at zero energy for long-range potentials. It is shown that if $V=-\alpha|x|^{-\mu}+W$ for some $\alpha>0$, $0<\mu<2$ and a sufficiently fast decaying, radial function $W$, then the scattering matrix for the pair $(-\Delta+V,-\Delta)$ at zero energy is given by

$$
\exp \left(-i \frac{\pi \mu}{2-\mu} \sqrt{-\Delta_{\mathbb{S}^{d-1}}+\left(\frac{d-2}{2}\right)^{2}}\right)+K
$$

for some compact operator $K$ (depending on $V$ ). Moreover, it is conjectured that if $V=-\alpha|x|^{-\mu}$ (that is, if $W \equiv 0$ ), then $K \equiv 0$. The purpose of this note is to prove this conjecture.

Theorem 1. Let $d \geq 2,0<\mu<2$ and $\alpha>0$. For $\lambda>0$ let $S(\lambda)$ be the scattering matrix for the pair $\left(-\Delta-\alpha|x|^{-\mu},-\Delta\right)$ at energy $\lambda$ in the sense of [DeSk1]. Then

$$
\mathrm{s}-\lim _{\lambda \rightarrow 0+} S(\lambda)=\exp \left(-i \frac{\pi \mu}{2-\mu} \sqrt{-\Delta_{\mathbb{S}^{d-1}}+\left(\frac{d-2}{2}\right)^{2}}\right) .
$$

It was shown in DeSk1 that $S(0):=\mathrm{s}-\lim _{\lambda \rightarrow 0+} S(\lambda)$ exists and is given through the phase shifts of the solutions of the energy zero equations corresponding to fixed angular momentum. To be more precise, for any $l \in \mathbb{N}_{0}$ there exists a function $f$ satisfying

$$
\begin{aligned}
-f^{\prime \prime}+\left(\frac{(l-1+d / 2)^{2}-1 / 4}{r^{2}}-\frac{\alpha}{r^{\mu}}\right) f & =0 \quad \text { in } \mathbb{R}_{+}, \\
\lim _{r \rightarrow 0} r^{-l-\frac{d-1}{2}} f(r) & =1, \\
\lim _{r \rightarrow \infty}\left(r^{-\mu / 4} f(r)-C_{l} \sin \left(\frac{2 \sqrt{\alpha}}{2-\mu} r^{\frac{2-\mu}{2}}+D_{l}\right)\right) & =0
\end{aligned}
$$

for some $C_{l}>0$, and in terms of this function the action of $S(0)$ on a spherical harmonic $Y$ of order $l$ is given by

$$
S(0) Y=\exp \left(i 2\left(D_{l}+\frac{\pi}{4}(d-3+2 l)\right)\right) Y .
$$

(Note that the $2 \pi$-ambiguity in the definition of $D_{l}$ does not affect the formula for $S(0)$.) In view of these facts and recalling that the Laplacian acts on spherical harmonics of order $l$ as multiplication by $l(l+d-2)$, Theorem 1 will follow if we can prove

(c) 2008 by the author. This paper may be reproduced, in its entirety, for non-commercial purposes. 
that $D_{l}=-\frac{\pi(d-2+2 l)}{2(2-\mu)}+\frac{\pi}{4}$ modulo $2 \pi$. This equality is the assertion of the following lemma applied to $\nu=l+\frac{d-2}{2}$.

Lemma 2. Let $\nu \geq 0,0<\mu<2$ and $\alpha>0$. The function

$$
f(r):=\Gamma\left(\frac{2 \nu}{2-\mu}+1\right)\left(\frac{2-\mu}{\sqrt{\alpha}}\right)^{\frac{2 \nu}{2-\mu}} r^{1 / 2} J_{\frac{2 \nu}{2-\mu}}\left(\frac{2 \sqrt{\alpha}}{2-\mu} r^{\frac{2-\mu}{2}}\right)
$$

satisfies

$$
\begin{aligned}
-f^{\prime \prime}+\left(\frac{\nu^{2}-1 / 4}{r^{2}}-\frac{\alpha}{r^{\mu}}\right) f & =0 \quad \text { in } \mathbb{R}_{+}, \\
\lim _{r \rightarrow 0} r^{-\nu-1 / 2} f(r) & =1, \\
\lim _{r \rightarrow \infty}\left(r^{-\mu / 4} f(r)-C \sin \left(\frac{2 \sqrt{\alpha}}{2-\mu} r^{\frac{2-\mu}{2}}-\frac{\pi \nu}{2-\mu}+\frac{\pi}{4}\right)\right) & =0
\end{aligned}
$$

with $C:=\pi^{-1 / 2} \Gamma\left(\frac{2 \nu}{2-\mu}+1\right)((2-\nu) / \sqrt{\alpha})^{\frac{2 \nu}{2-\mu}+\frac{1}{2}}>0$.

Proof. We will show that the function $f$ given in (1i) is the unique solution of the initial value problem (2) - (3). Then the asymptotics (44) follow from the asymptotics

$$
J_{\tilde{\nu}}(s)=\sqrt{\frac{2}{\pi s}}\left(\sin \left(s-\frac{\pi \tilde{\nu}}{2}+\frac{\pi}{4}\right)+o(1)\right), \quad s \rightarrow \infty,
$$

of Bessel functions [AbSt, (9.2.1)].

Let $f$ denote any solution of the initial value problem (2) - (3) and define $g$ by

$$
f(r)=: r^{1 / 2} g\left(b r^{\frac{2-\mu}{2}}\right), \quad b:=\frac{2 \sqrt{\alpha}}{2-\mu} .
$$

This definition is motivated by the asymptotics (44): If we want to arrive at a function which behaves asymptotically like an inverse square root times an oscillating function - which is the asymptotic behavior of any Bessel function -, then (4) suggests to look at $r^{-1 / 2} f(r)$ as a function of $r^{\frac{2-\mu}{2}}$. This is essentially what we call $g$, and we are about to show that $g$ is indeed a Bessel function.

A short computation shows that equation (2) in terms of $g$ becomes

$$
g^{\prime \prime}+s^{-1} g^{\prime}-\left(\frac{2 \nu}{2-\mu}\right)^{2} s^{-2} g+g=0,
$$

which is Bessel's equation with parameter $\tilde{\nu}:=2 \nu /(2-\mu)$. Hence $g$ is a linear combination of $J_{\tilde{\nu}}$ and $Y_{\tilde{\nu}}$. Boundary condition (3) in terms of $g$ becomes

$$
\lim _{s \rightarrow 0} s^{-\tilde{\nu}} g(s)=b^{-\tilde{\nu}} \text {. }
$$

Since $J_{\tilde{\nu}}(s) \sim(s / 2)^{\tilde{\nu}} / \Gamma(\tilde{\nu}+1)$ for all $\tilde{\nu} \geq 0$ and $Y_{\tilde{\nu}}(s) \sim-(1 / \pi) \Gamma(\tilde{\nu})(s / 2)^{-\tilde{\nu}}$ for $\tilde{\nu}>0$, resp. $Y_{0}(s) \sim(2 / \pi) \ln s$ as $s \rightarrow 0$ [AbSt, $\left.(9.1 .7-9)\right]$, we conclude that

$$
g(s)=\Gamma(\tilde{\nu}+1)(2 / b)^{\tilde{\nu}} J_{\tilde{\nu}}(s) .
$$


This proves the lemma.

Acknowledgements. The author wishes to thank R. Seiringer for useful discussions. Support through DFG grant FR 2664/1-1 and U.S. NSF grant PHY 0652854 is gratefully acknowledged.

\section{REFERENCES}

[AbSt] M. Abramowitz, I. A. Stegun, Handbook of mathematical functions with formulas, graphs, and mathematical tables. Reprint of the 1972 edition. Dover Publications, New York, 1992.

[DeSk1] J. Dereziński, E. Skibsted, Quantum scattering at low energies. Preprint (2008).

[DeSk2] J. Dereziński, E. Skibsted, Scattering at zero energy for attractive homogeneous potentials. Preprint (2008).

Rupert L. Frank, Department of Mathematics, Princeton University, Washington RoAd, Princeton, NJ 08544, USA

E-mail address: rlfrank@math.princeton.edu 